\title{
Interferometric studies of laser-created plasmas using compact soft x-ray lasers
}

J. Filevich, J.J. Rocca, E.C. Hammarsten, E. Jankowska, M.C. Marconi; Colorado State University: R.F. Smith, R. Keenan, J. Dunn, S. Moon, J. Nilsen, J.R. Hunter; Lawrence Livermore National Laboratory: V. Shlyaptsev; University of California Davis-Livermore

This article was submitted to SPIE 2003 Conference

\section{December 2003}

U.S. Department of Energy

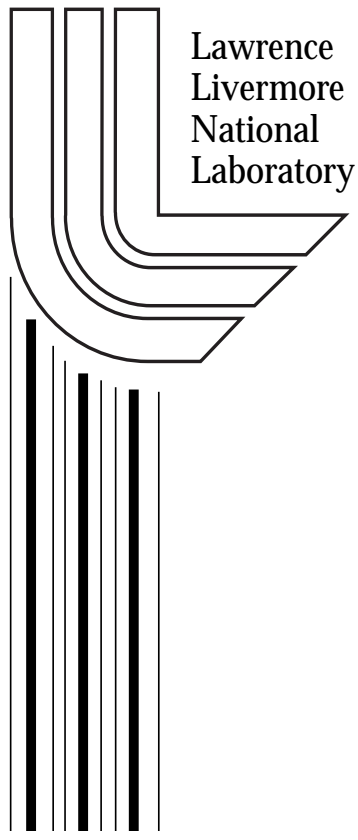




\section{DISCLAIMER}

This document was prepared as an account of work sponsored by an agency of the United States Government. Neither the United States Government nor the University of California nor any of their employees, makes any warranty, express or implied, or assumes any legal liability or responsibility for the accuracy, completeness, or usefulness of any information, apparatus, product, or process disclosed, or represents that its use would not infringe privately owned rights. Reference herein to any specific commercial product, process, or service by trade name, trademark, manufacturer, or otherwise, does not necessarily constitute or imply its endorsement, recommendation, or favoring by the United States Government or the University of California. The views and opinions of authors expressed herein do not necessarily state or reflect those of the United States Government or the University of California, and shall not be used for advertising or product endorsement purposes.

This is a preprint of a paper intended for publication in a journal or proceedings. Since changes may be made before publication, this preprint is made available with the understanding that it will not be cited or reproduced without the permission of the author. 


\title{
Interferometric studies of laser-created plasmas using compact soft $x$-ray lasers.
}

\author{
Jorge Filevich ${ }^{1}$, Jorge J. Rocca ${ }^{1}$, Eric C. Hammarsten ${ }^{1}$, Elzbieta Jankowska ${ }^{1}$, Mario C. \\ Marconi $^{1,2}$,Raymond F. Smith ${ }^{3}$, Roisin Keenan, James Dunn ${ }^{3}$, Stephen Moon ${ }^{3}$, \\ Vyacheslav Shlyaptsev ${ }^{4}$, Joseph Nilsen ${ }^{3}$, and James R. Hunter ${ }^{3}$ \\ ${ }^{I}$ Dept. of Electrical and Computer Engineering, Colorado State University, Fort Collins, CO 80523 \\ ${ }^{2}$ Dept. of Physics, University of Buenos Aires, Argentina. \\ ${ }^{3}$ Lawrence Livermore National Laboratory, Livermore, CA 94551 \\ ${ }^{4}$ Department of Applied Science, University of California Davis-Livermore, Livermore, CA 94551
}

\begin{abstract}
We summarize results of several successful dense plasma diagnostics experiments realized by combining two different kinds of table-top soft $\mathrm{x}$-ray lasers with an amplitude division interferometer based on diffraction grating beam splitters. In the first set of experiments this robust high throughput diffraction grating interferometer (DGI) was used with a $46.9 \mathrm{~nm}$ portable capillary discharge laser to study the dynamics of line focus and point focus laser-created plasmas. The measured electron density profiles, which differ significantly from those expected from a classical expansion, unveil important two-dimensional effects of the dynamics of these plasmas. A second DGI customized to operate in combination with a $14.7 \mathrm{~nm}$ Ni-like Pd transient gain laser was used to perform interferometry of line focus laser-created plasmas with picosecond time resolution. These measurements provide valuable new benchmarks for complex hydrodynamic codes and help bring new understanding of the dynamics of dense plasmas. The instrumentation and methodology we describe is scalable to significantly shorter wavelengths, and constitutes a promising scheme for extending interferometry to the study of very dense plasmas such as those investigated for inertial confinement fusion.
\end{abstract}

Keywords: soft x-ray laser, soft x-ray interferometry, laser-created plasmas.

\section{Introduction.}

Soft $\mathrm{x}$-ray interferometry was first demonstrated using laboratory size $\mathrm{x}$-ray lasers [1,2]. This previous work succeeded in demonstrating its feasibility as a powerful technique for the diagnostics of dense large-scale plasmas. However, it suffered from the limitations in repetition rate, complexity, and cost associated with the original laboratory size x-ray lasers, which limited most of the studies to a few shots per day. The feasibility of conducting plasma interferometry with a table-top laser was first demonstrated with a compact capillary discharge soft x-ray laser and a Lloyd's mirror interferometer [3,4]. The results presented here demonstrate that currently available compact soft x-ray lasers with spectral brightness that match or exceed that of their laboratory-size predecessors constitute unique tools for the diagnostics of dense plasmas. Their high repetition frequency and versatility allows extensive systematic studies of the dynamics of dense large-scale plasmas. As an illustration, in Fig 1. it is shown how the short wavelength of these compact lasers extends the plasma parameter space accessible for probing significantly beyond the limits of optical interferometry. The limits are computed based on refraction considerations, which is normally the strongest limitation. As an illustration of the dramatic reduction of refraction achieved by reducing the wavelength of the probe Fig. 2 compares the computed trajectories of a $335 \mathrm{~nm}$ beam ( $3^{\text {rd }}$ harmonic of Nd:YAG) and a $46.9 \mathrm{~nm}$ Ne-like Ar capillary discharge laser traveling through a line focus plasma. The density gradient responsible for the beam deflection was created irradiating a $\mathrm{Cu}$ target with a laser intensity of $0.1 \mathrm{TW} \mathrm{cm}^{-2}$

In these proceedings we present results obtained combining a robust high throughput amplitude division interferometer with two different x-ray lasers. Two slightly modified versions of the same diffraction grating interferometer were used with a portable capillary discharge $46.9 \mathrm{~nm}$ laser and a picosecond resolution $14.7 \mathrm{~nm}$ transient collisional laser (TCE). The potential of this technique is demonstrated by the large amount of data obtained 
from laser-created plasmas for a broad range of target irradiation conditions.

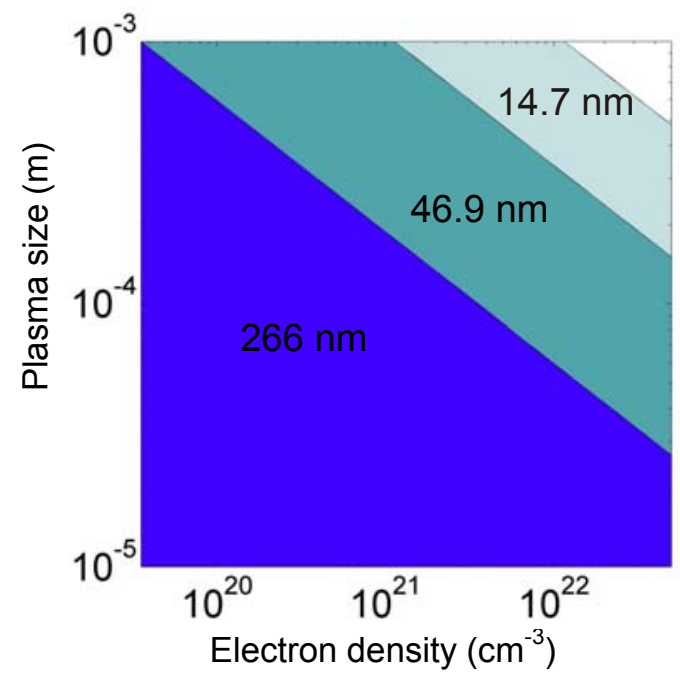

Figure 1. Plasma parameter space accessible to plasma interferometry with a) $4^{\text {th }}$ harmonic of Nd-YAG, b) $46.9 \mathrm{~nm}$ portable soft $x$-ray laser, c) $14.7 \mathrm{~nm}$ Ni-like Pd soft $x$-ray laser. The limits shown are determined from refraction considerations. (The relation between the plasma size and the electron density for a given wavelength is obtained from the refraction length: $L_{r}=\frac{d}{2} \sqrt{\frac{n_{c}}{n_{e}}} \propto \frac{d}{\lambda \sqrt{n_{e}}}$ where $\mathbf{d}$ is the lateral size of the plasma).

In the next section we present a detailed description of the common instrument used in these studies: the diffraction grating interferometer (DGI) that has proven to be a versatile and robust tool for plasma diagnostics. It's design has the additional advantage of allowing operation at any of the wavelengths presently produced by soft x-ray lasers, and is also scalable to shorter wavelengths. Section III summarizes the results obtained with a portable $46.9 \mathrm{~nm}$ capillary discharge laser, and section IV gives examples of the results obtained with a $14.7 \mathrm{~nm}$ transient gain laser.

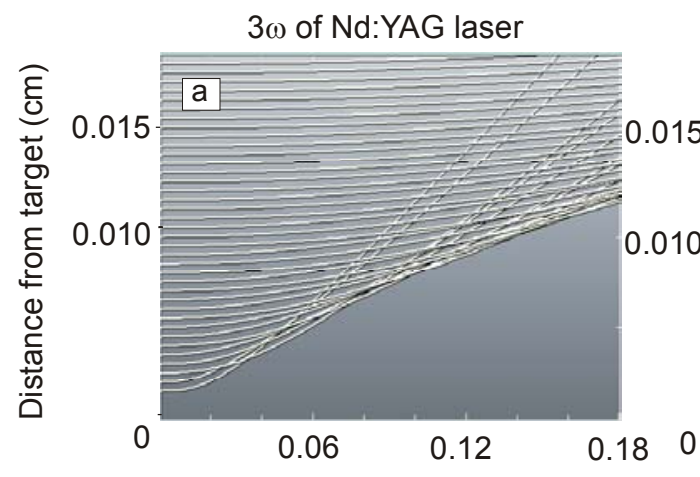

Distance along target $(\mathrm{cm})$

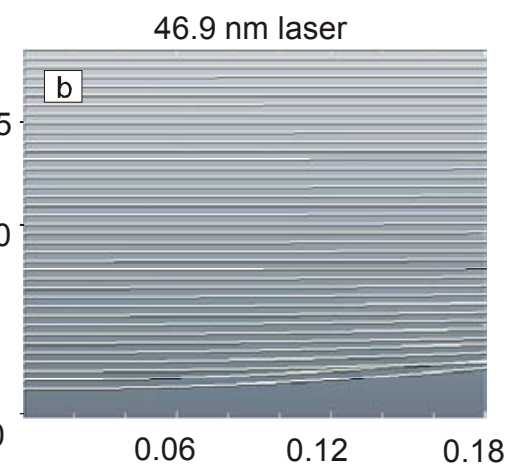

Distance along target $(\mathrm{cm})$

Figure 2. Refraction simulation for a probe beam as it goes through a plasma, comparing for two different wavelengths. Within the region $100 \mu \mathrm{m}$ away from the target the $3 \omega$ of the Nd:YAG laser is completely refracted away from its original path while the $46.9 \mathrm{~nm}$ laser is practically unaffected by refraction. The computations were performed using the code RADEX [5]. 


\section{II.Diffraction Grating Interferometer.}

The amplitude division interferometer we have developed uses reflective diffraction gratings as beam splitters[6,7] in a grazing Mach-Zehnder configuration. The use of diffraction gratings allows the construction of an interferometer of increased robustness and high throughput that operates over an extended range of soft x-ray wavelengths. The diffraction grating interferometer (DGI) is set in a skewed Mach-Zehnder configuration as shown in Figure 3. The light incident on the first grating (right of the figure) is diffracted with approximately equal intensity onto the zero and first orders. These two beams are reflected towards a second diffraction grating using a pair of $35 \mathrm{~cm}$ long Au-coated mirrors, forming the two arms of the interferometer. The second grating recombines the two beams such that they exit the interferometer propagating with a small angular difference selected to produce fringes of the spacing required by the particular experiment.

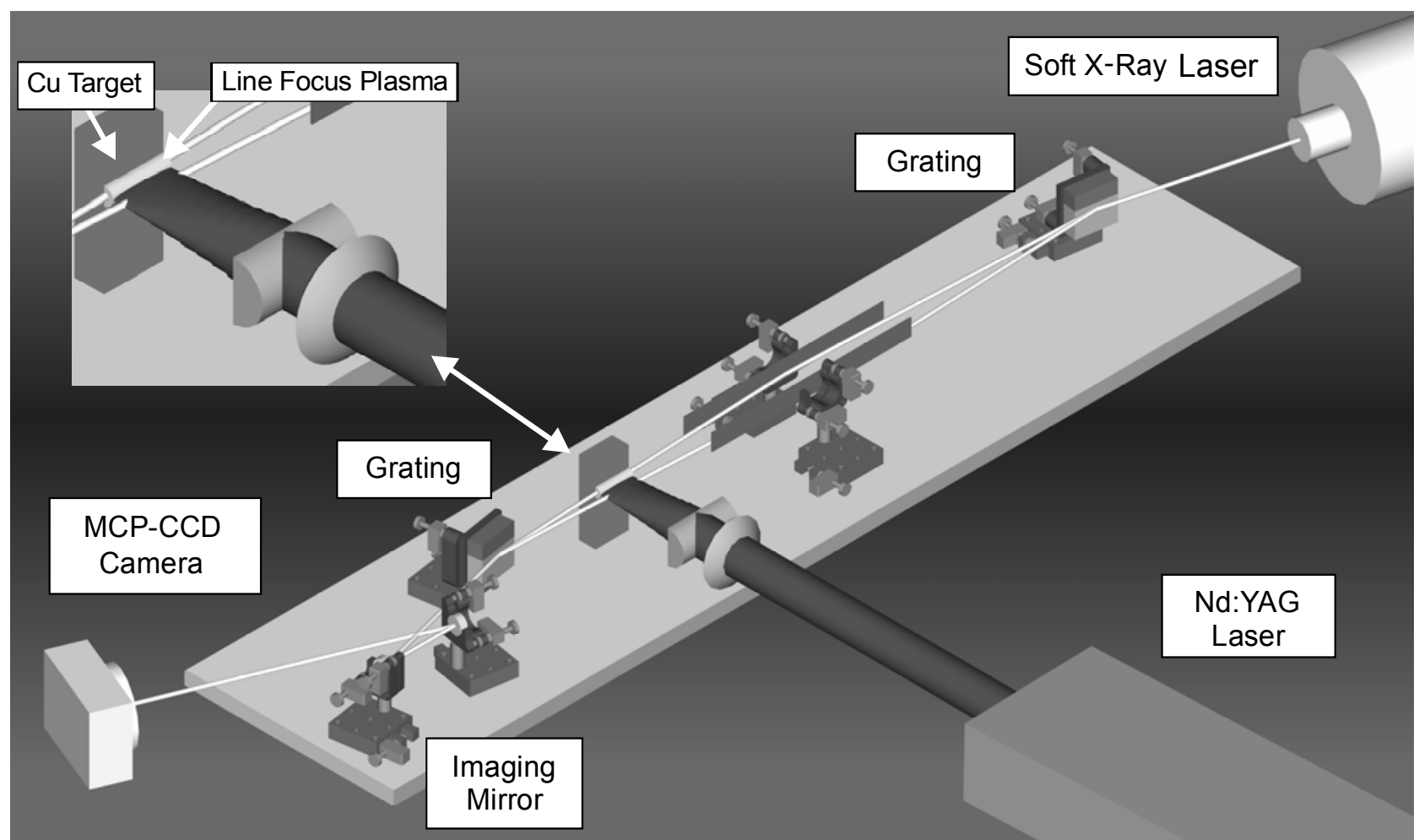

Figure 3: Diffraction Grating Interferometer as used for a line focus plasma probing experiment using a $46.9 \mathrm{~nm}$ capillary discharge laser probe.

Two versions of the interferometer were constructed, the first one, successfully implemented at Colorado State University together with the $46.9 \mathrm{~nm}$ capillary discharge soft x-ray laser, uses gratings with $300 \mathrm{l} / \mathrm{mm}$ and $1.3 \mathrm{degrees}$ blaze angle. The second version, constructed to operate in combination with a $14.7 \mathrm{~nm}$ TCE soft x-ray laser uses gratings with $900 \mathrm{l} / \mathrm{mm}$ and a 2 degrees blaze angle. This difference in grating characteristics is the most significant difference between the two versions of the DGIs, and is the only modification required to tailor the interferometer to any of the currently available soft x-ray laser wavelengths. The two gratings designs split the beam into a zero and a first order beam of approximately the same intensity when operating at an incidence angle of 11 and 6 degrees respectively. The reflectivity of the gold-coated diffraction gratings is maximized by the small grazing incidence angle used, resulting in an efficiency of $25 \%$ per order on each grating. The long mirror's reflectivity is $\sim 90 \%$, giving a total throughput for the instrument of $\sim 12 \%$. The initial alignment of the interferometer is done with an auxiliary IR alignment diode. To allow the IR laser and the soft x-ray beams to follow the same path an auxiliary ruling of the appropriate line density is manufactured on the same substrate. The IR laser diodes are chosen to have a temporal coherence length similar or shorter than that of the soft x-ray laser ( 400 microns 1/e HW [8] for the $14.7 \mathrm{~nm}$ laser and an estimated $\sim 250 \mathrm{microns}$ 
for the $46.9 \mathrm{~nm}$ laser [7]). Therefore, generating fringes with the alignment diode ensures that the arm lengths are matched with sufficient precision to warrant the observation of fringes with the soft $\mathrm{x}$-ray laser. The plasma, generated within the zero order arm of the interferometer, is imaged onto a two dimensional detector with a magnification that can be changed between typically $10 \mathrm{X}$ and $50 \mathrm{X}$. The imaging is accomplished with multilayer-coated spherical mirrors, set at an incidence angle of 5 degrees or less to reduce astigmatism. The spatial resolution was found to be $\sim 2$ micrometers for the system using a $12.4 \mu \mathrm{m}$ pixel size CCD camera. The alignment procedure is similar for both interferometers where the zero order path is aligned first with a visible laser. Subsequently the first order is aligned using the IR laser diode and the auxiliary ruling on the gratings, until fringes are observed. The visible laser is used also to steer the output beam through the imaging optics onto the detector, using the zero order arm. As the optimum alignment can differ slightly from that obtained with the IR beam the final adjustments are conducted under vacuum with the soft x-ray laser beam, utilizing motorized actuators to control the position of the relevant optics.

\section{Interferometry with a portable capillary discharge soft x-ray laser}

The first demonstration of the DGI was conducted using a $46.9 \mathrm{~nm}$ Ne-like Ar capillary discharge soft x-ray laser, and consisted of the study of a line-focus plasma generated with $1 \omega(1054 \mathrm{~nm}) \mathrm{Nd}$-YAG laser intensities of about $0.034 \mathrm{TW} / \mathrm{cm}^{2}$. Those measurements showed that the plasma evolution displayed the expected classical expansion [7]. The results presented in this section correspond to plasmas generated at increased intensities of 0.1 to $7 \mathrm{TW} / \mathrm{cm}^{2}$. To generate the line foci plasmas, the pump laser was focused into a $30 \mu \mathrm{m}$ wide line $1.8 \mathrm{~mm}$ in length using the combination of a cylindrical and a spherical lens. The resulting line focus intensity distribution corresponding to the experiments conducted for a target irradiation intensity of $0.1 \mathrm{TW} \mathrm{cm}^{-2}$ is shown in figure 4 .

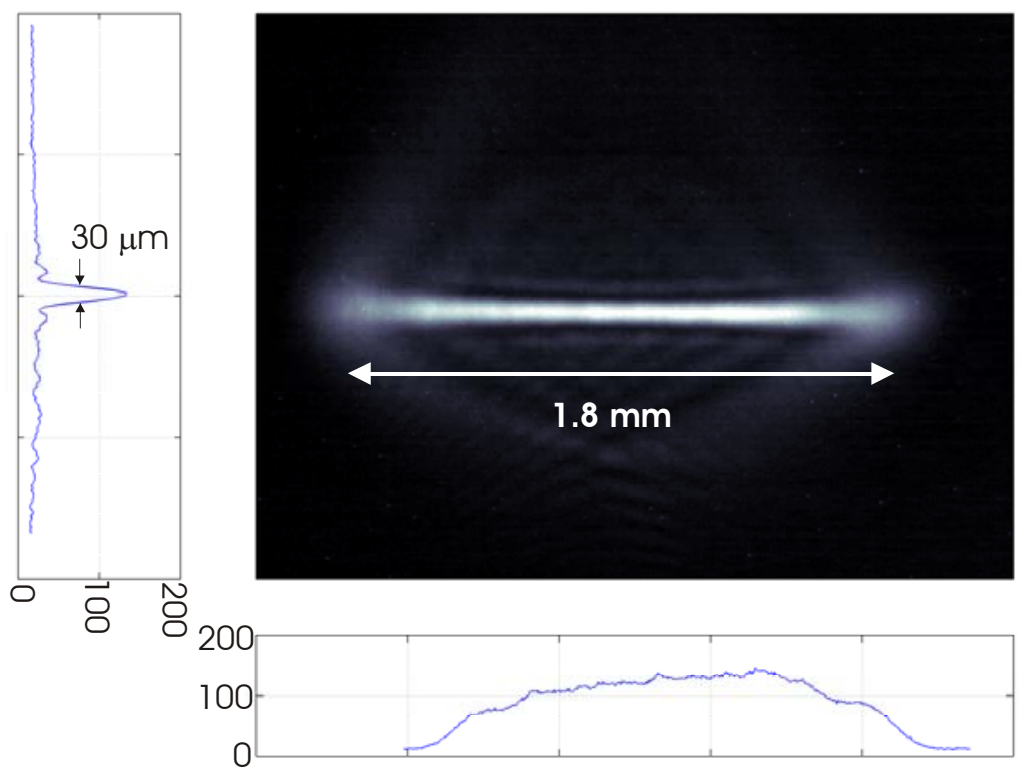

Figure 4. Intensity distribution, measured at the target position, of the heating beam used to create the line focus plasma studied with $46.9 \mathrm{~nm}$ laser interferometry.

A series of interferograms was obtained to map the evolution of the plasma. The earlier interferograms show an expanding plasma with a density distribution that presents a maximum on axis, as was the case with measurements at lower irradiation intensities. However, as time evolves and the pump laser intensity increases, the plasma density profile acquires a concave shape, with minimum density on axis. Figure 5 (left) shows an interferogram where the density depression is clearly visible. This interferogram was taken at $12 \mathrm{~ns}$ after the initiation of the heating laser pulse which had $\sim 13$ ns FWHM duration. The electron density profile constructed from the fringe shift map is shown in figure 3 (center). The observed formation of this curious concave electron density profile is reproduced well by hydrodynamic model simulations conducted with LASNEX (Fig. 5 right), and is discussed at the end of this section. 

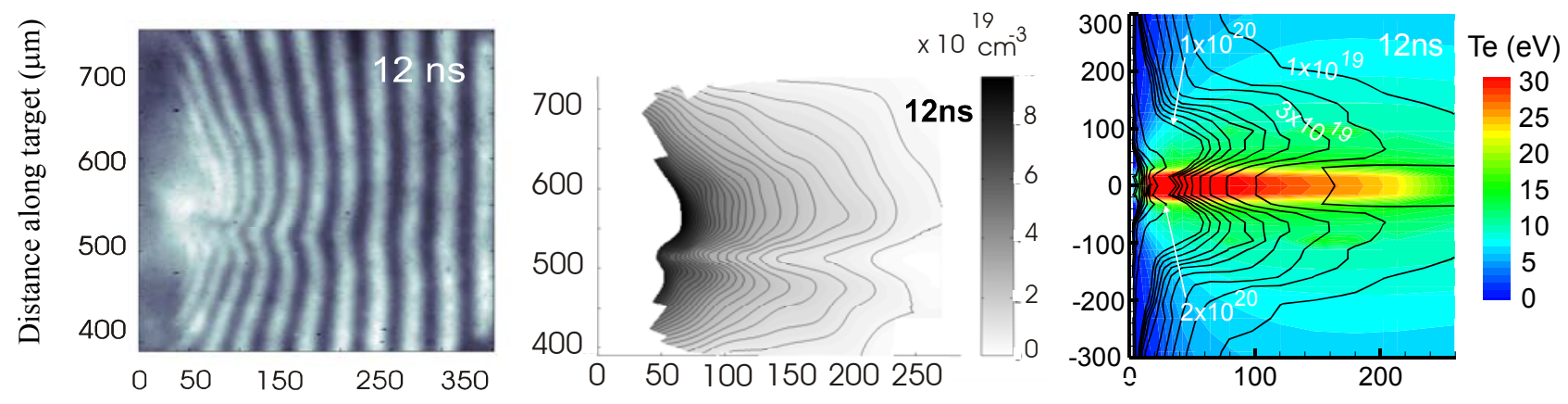

Distance from target $(\mu \mathrm{m})$

Figure 5. Interferogram of a $\mathrm{Cu}$ line focus laser created plasma that shows an on-axis density depression (left). Electron density obtained from that interferogram (center). LASNEX simulation that reproduces the experimental results (right).

Plasmas created at larger irradiation intensities $\left(7 \mathrm{TW} \mathrm{cm}^{-2}\right)$ were generated by focusing $0.62 \mathrm{~J}$ pulses from a $\mathrm{Nd}$ :YAG laser onto a spot $<30 \mu \mathrm{m}$ in diameter on a $\mathrm{Cu}$ target using a $15 \mathrm{~cm}$ focal length aspheric lens. In this case the plasmas were imaged with $51 \mathrm{X}$ magnification. Series of interferograms were obtained for plasmas generated by either firing the laser onto a new target spot, or after firing several laser shots in the same target location.
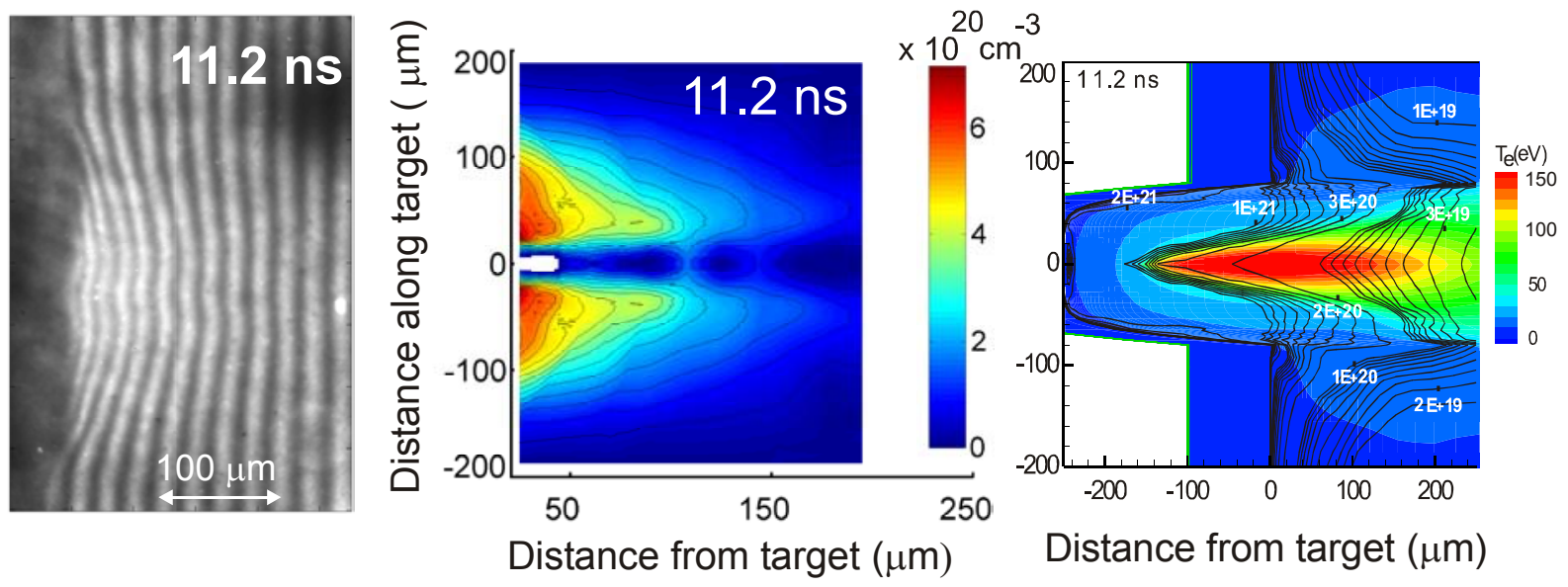

Figure 6. Interferogram of a $\mathrm{Cu}$ point-focus plasmas generated by a second laser shot in the same target location (left). Electron density obtained after an Abel inversion from that interferogram (center). LASNEX simulation that reproduces the experimental results (right).

The plasmas produced by firing multiple laser shots in the same target location are observed to extend over a significantly larger volume. This is a result of the fact that these plasmas emanate from the crater created by the previous shots, which guides the plasma expansion generating large plasma densities at distances more than hundred micrometers from the target.

The Nd-YAG laser that generates the plasma was focused into a spot with an intensity of $\sim 7 \mathrm{TW} / \mathrm{cm}^{2}$. Time was measured with respect to the beginning of the heating laser pulse. The electron density distributions resulting from Abel inversion of the interferogram is shown in figure 6 (center). The interesting formation of a concave electron density profile is again observed. In the region mapped by the interferograms the electron density is observed to increase as a function of time, to reach a maximum value of $9 \times 10^{20} \mathrm{~cm}^{-3}$ at 27 micrometers from the target near the time of maximum laser intensity. This value amounts to about $90 \%$ of the critical density for the $\lambda=1.06 \mu \mathrm{m}$ pump laser. As in the case of the line-focused plasmas a density minima is observed to develop on axis. At the time of maximum laser intensity this central minimum in the density profile is observed to extend through practically the entire sub-critical region of the 
plasma. It should be noticed that the density minima is not a consequence of the crater created by the laser pulse, as it was also observed on first shots on a new target.

\section{Comparison with hydrodynamic model simulations.}

Simulations of the measured plasmas conducted using LASNEX [9] successfully reproduced the observations and helped to understand the origin of the axial density depression. The computations for both the line focus and spot focus plasmas show the formation of the concave electron profile observed in the experiments. In agreement with the measured density profiles of figures 5 and 6 , the density distribution of the line focus plasma is computed to evolve from a convex profile, during the first few ns of the expansion, into a concave density profile, with the central depression starting about $4 \mathrm{~ns}$ after the initiation of the laser pulse. The computed density profiles (right of Figs. 5 and 6 ) show the same predominant side-lobes and density minima observed in the interferograms. The modeling rules out the ponderomotive force and heating of the plasma far away from the surface as the main causes of the concave electron density profile. Instead, it points to hydrodynamic and radiation effects as the primary reasons. The resulting physical picture consists of a central high temperature $(\sim 30 \mathrm{eV})$ region surrounded by a lower temperature $(\sim 10 \mathrm{eV})$ plasma on either side. The on-axis density distribution and velocity fit well the results of 1D and 2D simulations of a laser irradiated plasma. Instead, the side-lobes are a 2-D effect formed outside the laser irradiated target region by the expansion of colder material that originates from an ablation area that is enlarged by a factor 3-4 by heat conduction and radiation emanating from the hot focal spot. From the $2 \mathrm{D}$ simulations it is clear that the effect that we observed is due to the combination of the build up of the high density lower temperature side-lobes and the depletion of the density in the higher temperature on-axis plasma. The heating of the central portion of the plasma yields a higher temperature, and large pressure gradients near the critical surface that result in larger axial velocity that causes the density along the center line to blow out faster $\left(5-6 \times 10^{6} \mathrm{~cm} \mathrm{~s}^{-1}\right)$ than the slower higher density side-lobes. Since this central region ejects its mass on a time scale that is fast compared to the expansion of the side-lobes the constant density contour develop a density minimum on axis, which is an inversion of the expected result.

Simulations conducted for the spot focus plasmas also show the formation of concave electron density with a minimum on axis [10]. As is also the case for the line focus experiment, the simulation shows that the density of the sidelobe is significantly increased by plasma radiation-induced ablation of target material from the area surrounding the laser irradiated spot. Plasma radiation is also a major cooling mechanism for the sidelobe plasma. Pressure balance between the two concentric regions contributes to the development of a density depression on axis, similarly to the previously studied case of a line-focus plasma [11]. However, the absence of radiation does not stop the 'hole formation' in the case of deep crater-based plasmas. Across such relatively narrow crater pressure balance easily takes place, hence creating a density depression along the laser-irradiated axis. In other words, the laser pulse is sufficiently long for the interior of the of crater to be filled with plasma ablated from the focal spot at the crater's bottom and for sound waves to transverse the crater dimension multiple times. In addition, in the present case the crater constrains the plasma expansion in the lateral direction and guides the motion in the direction perpendicular to the target, enhancing the density at large distances from the surface. Plasma radiation adds additional ablated mass, significantly enhancing the magnitude of the plasma density and the depression outside the cavity. The results demonstrate that table-top lasers are a powerful tool for the diagnostics of dense plasmas, that in combination with hydrodynamic codes can result in improved understanding of high density plasma phenomena.

\section{Picosecond Resolution Plasma Probing}

The interferometric diagnostics of the denser, more rapidly evolving regions of laser-created plasmas require picosecond resolution to overcome the blurring of interference fringes associated with the rapidly changing densities. To realize the first demonstration of soft x-ray laser interferometry with picosecond resolution the DGI was combined with a transient $14.7 \mathrm{~nm} \mathrm{Ni-like} \mathrm{Pd} \mathrm{soft} \mathrm{x-ray} \mathrm{laser} \mathrm{[12].} \mathrm{The} \mathrm{soft} \mathrm{X-ray} \mathrm{laser} \mathrm{was} \mathrm{pumped} \mathrm{by} \mathrm{two} \mathrm{beams} \mathrm{from} \mathrm{a} \mathrm{chirped} \mathrm{pulse}$ amplification laser, the Compact Multipulse Terawatt (COMET) system at Lawrence Livermore National Laboratory that operates at $1054 \mathrm{~nm}$. A soft x-ray laser output of a few 10's of $\mu \mathrm{J}$ was achieved by optically pumping a polished Pd target with a sequence of a $600 \mathrm{ps}$ long pulse $\left(2 \mathrm{~J}, 2 \times 10^{11} \mathrm{~W} \mathrm{~cm}^{-2}\right)$ and a 13 ps short pulse $\left(5 \mathrm{~J}, 3 \times 10^{13} \mathrm{~W} \mathrm{~cm}^{-2}\right)$. Traveling wave line focus excitation was achieved using a reflection echelon that consists of seven flat mirror segments placed before the focusing optics. The divergence of the soft x-ray laser was measured to be $2.8 \mathrm{mrad}$. The near field and 
far field beam characteristics were studied and the laser parameters were optimized to obtain beam properties suitable to perform interferometry.

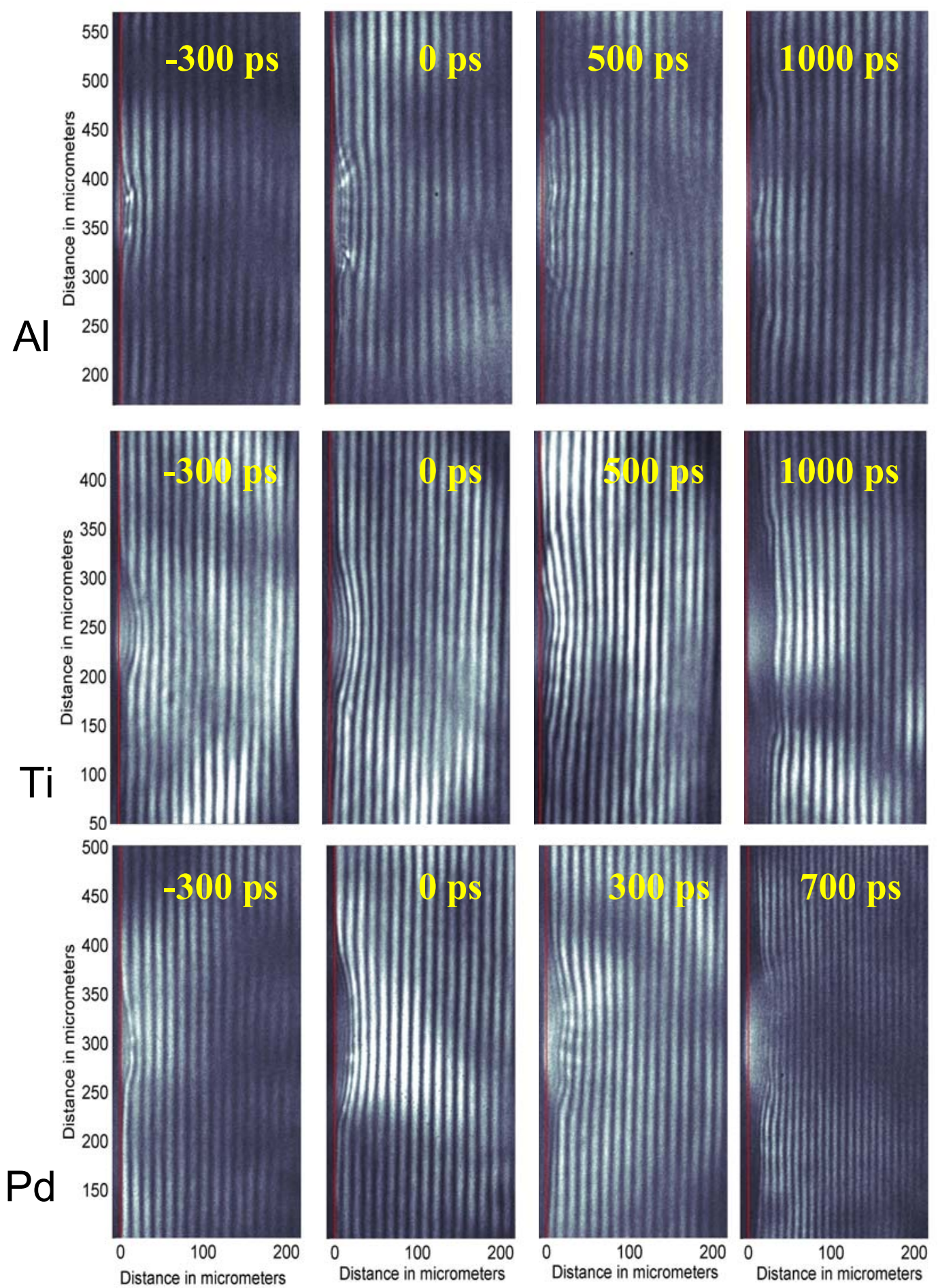

Figure 7: Sequences of $14.7 \mathrm{~nm}$ interferograms depicting the evolution of $1 \mathrm{~mm}$ long line focus laser-created plasmas generated heating slab targets of $\mathrm{Al}, \mathrm{Pd}$ and Ti with an intensity of $\sim 2.5 \mathrm{TW} / \mathrm{cm}^{2}$. The time shown is measured respect to the peak of the 600 ps heating pulse. 
The short soft x-ray laser pulse duration, of $\sim 5$ ps permits the acquisition of "snap-shots" of the rapidly evolving plasma, overcoming the blurring of interference fringes that may occur when the electron density profile changes significantly during the duration of the probe pulse. The combination of the robust grating interferometer with this laser capable of firing 1 shot every 4 minutes permitted the acquisition of sequences of interferograms that map the evolution of the electron density distribution in high density laser created plasmas. What follows is a summary of recent results that illustrate the possibilities of new studies opened by this powerful technique.

Figure 7 shows a series of interferograms that describe the evolution of plasmas generated by focusing $1 \mathrm{~J} 1 \omega$ $(1054 \mathrm{~nm})$ heating pulse of $600 \mathrm{ps}$ duration onto on a slab target of different materials $(\mathrm{Al}, \mathrm{Ti}, \mathrm{Pd})$ to produce a line focus $\sim 20 \mu \mathrm{m}$ wide and $3.2 \mathrm{~mm}$ long. The $1 \mathrm{~mm}$ long targets were overfilled to aid the generation of a uniform plasma. The size and homogeneity of the line focus were measured by imaging the plasma heating beam at the target plane with a through imaging system consisting of an achromatic lens and a CCD camera. The interferograms show that the plasma expansion evolution has a very significant component along the target surface direction, a characteristic that emphasizes its two-dimensional nature. Figure 8 shows the calculated density maps obtained from the interferograms in Fig 7 (Ti). Interferograms obtained at delays corresponding to the peak of the heating laser pulse and $500 \mathrm{ps}$ afterwards show a maximum density of $\sim 4.5 \times 10^{20} \mathrm{~cm}^{-3}$.
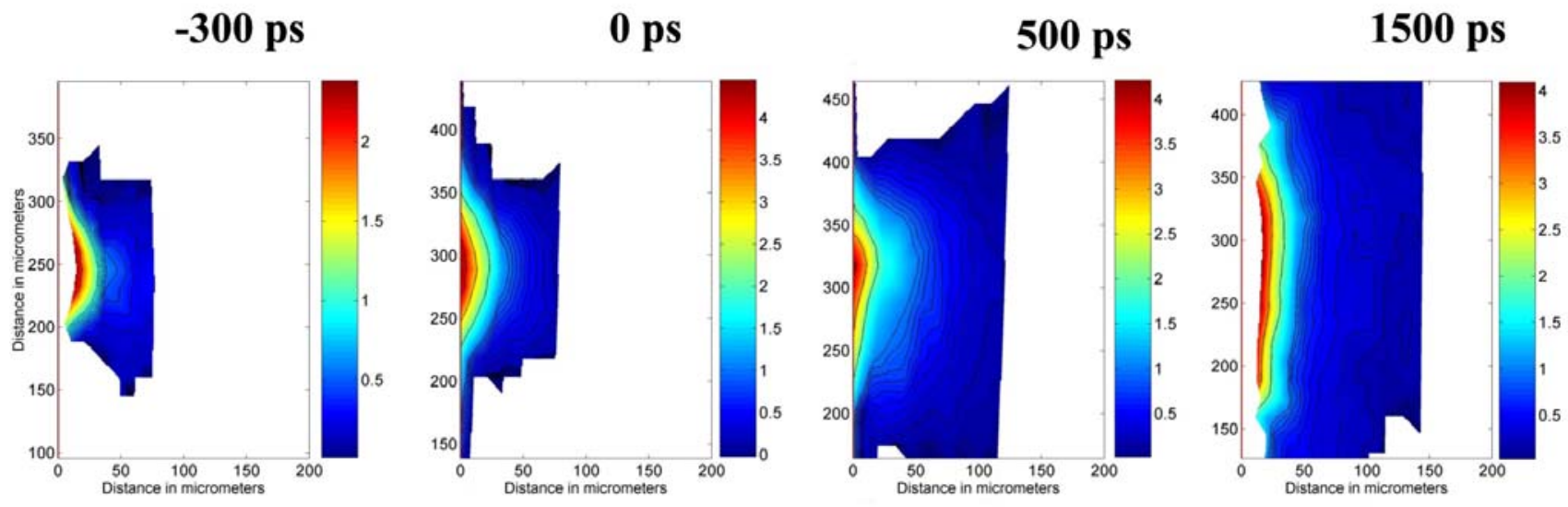

Figure 8. Electron density maps obtained from the Titanium series of interferograms of figure 7.

The large density gradients encountered in laser-created plasmas close to the target can produce significant steering of the probe beam away from the high-density regions. Knowledge of the extent and effect of refraction is therefore an important consideration for accurate analysis of the data. A grid reflectometry study was performed with the DGI [13] to estimate these refraction effects in $1 \mathrm{~mm}$ long plasmas such as those investigated herein. That study showed that the present data could be analyzed without the need of a correction due to refraction. This is in contrast with the use of an ultraviolet laser probe (e.g. the third harmonic of YAG, $267 \mathrm{~nm}$ ), whose beam would have been strongly refracted away from the region close to the target shortly after entering the $1 \mathrm{~mm}$ long plasma, making the probing of that region unfeasible.

Figure 9 shows a series of interferograms corresponding to plasmas obtained by irradiating flat $\mathrm{Al}$ target, increasing the intensity of the heating pulse to $\sim 13 \mathrm{TW} / \mathrm{cm}^{2}$. A density depression is again observed in this case. Figure 10 shows the electron density obtained from these interferograms. Numerical simulations are required to determine if the origin of this density depression is associated with the same physics responsible for the effects previously observed in plasmas created by longer heating pulses with the capillary discharge soft x-ray laser. 

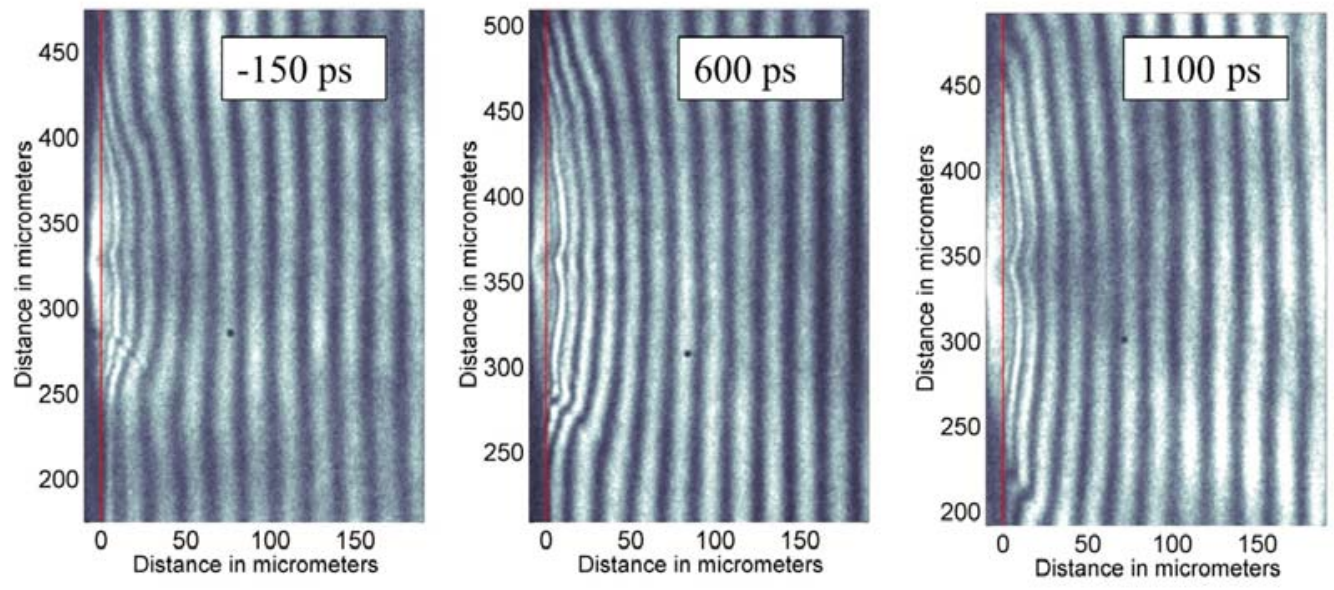

Figure 9. Series of interferograms depicting the evolution of a $12 \mu \mathrm{m}$ wide, $1 \mathrm{~mm}$ long line focus Al laser created plasma, where an on axis density depression is evident. The times are measured from the peak of the $600 \mathrm{ps,} 1 \mathrm{~J}$ heating pulse.
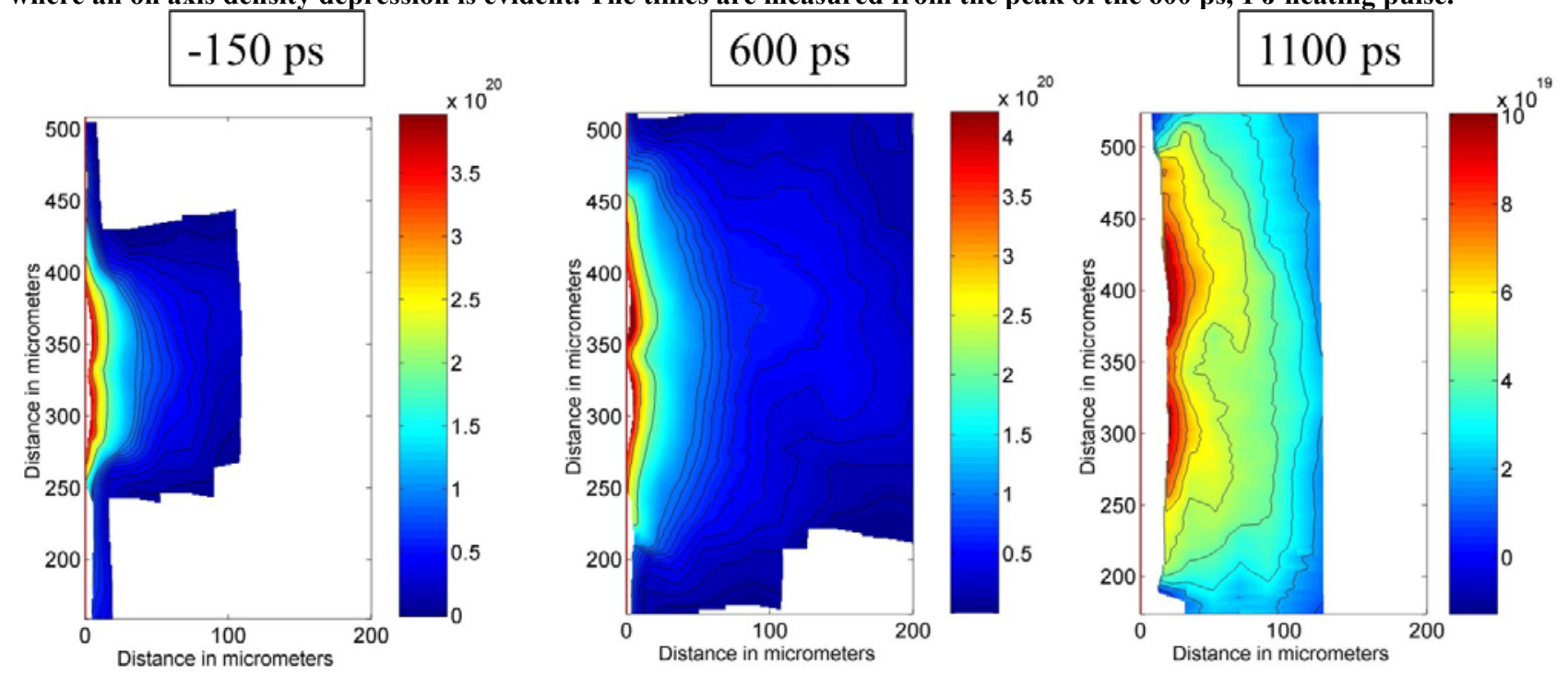

Figure 10. Electron density obtained from the series of interferograms shown in figure 9. The on axis depression is present in all the shots. The density reaches values of over $4 \times 10^{20} \mathrm{~cm}^{-3}$.

Another different type of targets investigated was a groove in aluminum $100 \mu \mathrm{m}$ wide and $200 \mu \mathrm{m}$ deep as shown in the top-left reference interferogram in Fig. 11. A third beam of the COMET laser was used to heat the bottom of the groove with a $3 \mathrm{~J}$ pulse focused in a tight $\sim 12 \mu \mathrm{m}$ line. The shots shown in Fig 10 (b) illustrate a sequence of interferograms obtained at different times from the peak of the heating laser pulse. 

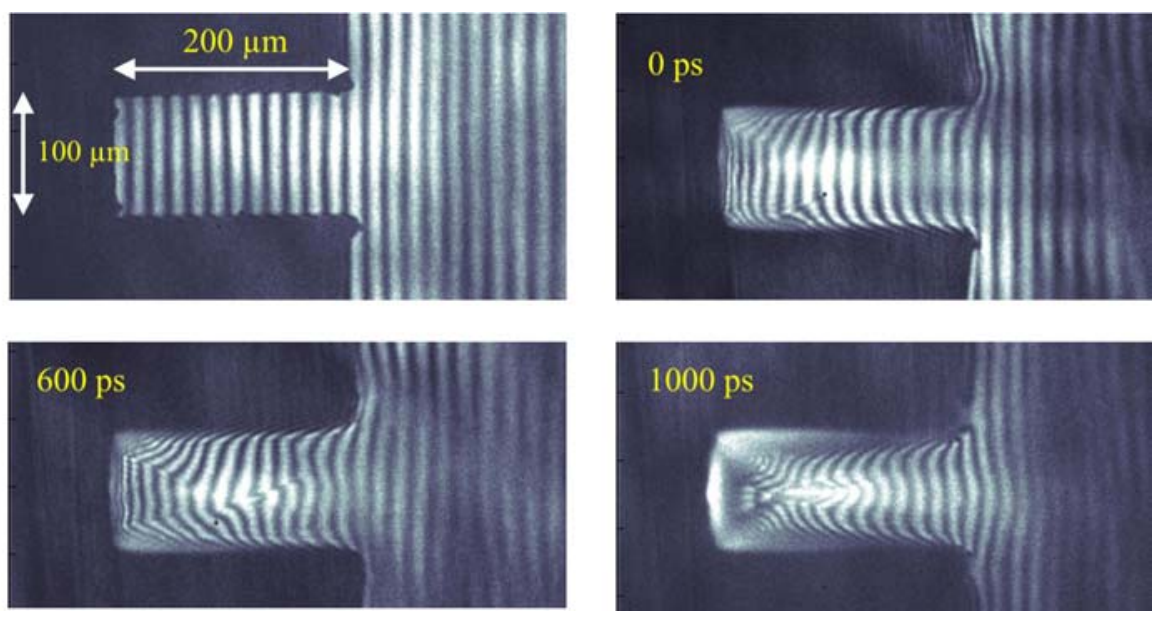

Figure 11. Sequence of interferograms corresponding to a plasma created by irradiating the bottom of a $1 \mathrm{~mm}$ long Al groove target with a $1 \mathrm{~J}, 1 \omega(1054 \mathrm{~nm}), 600$ ps laser pulse focused to a $12 \mu \mathrm{m}$ wide line focus. The top left frame shows an interferogram without plasma present, and the rest correspond to different times during the plasma evolution. Times are measured respect to the peak of the heating laser pulse.

The evolution of the plasma inside the groove has very different characteristics from that corresponding to a flat target. The very large and rapid lateral expansion of the plasma is constrained by the groove walls, forcing the plasma towards the center of the groove. The local maximum on axis can be interpreted as the collision of converging plasmas arising from the walls. The electron density map in Fig 12, shows an enhancement of the density on axis. The maximum density measured corresponds to the critical density for the heating laser pulse $\left(1 \times 10^{21} \mathrm{~cm}^{-3}\right)$. This example illustrates how picosecond soft $\mathrm{x}$-ray laser probes create the opportunities to study collisions and stagnation in large scale plasmas. A more complete study of the evolution of these plasmas will be published separately. Other results obtained with picosecond $14.7 \mathrm{~nm}$ interferometry are discussed in the paper by R. Smith et al in these proceedings [14]

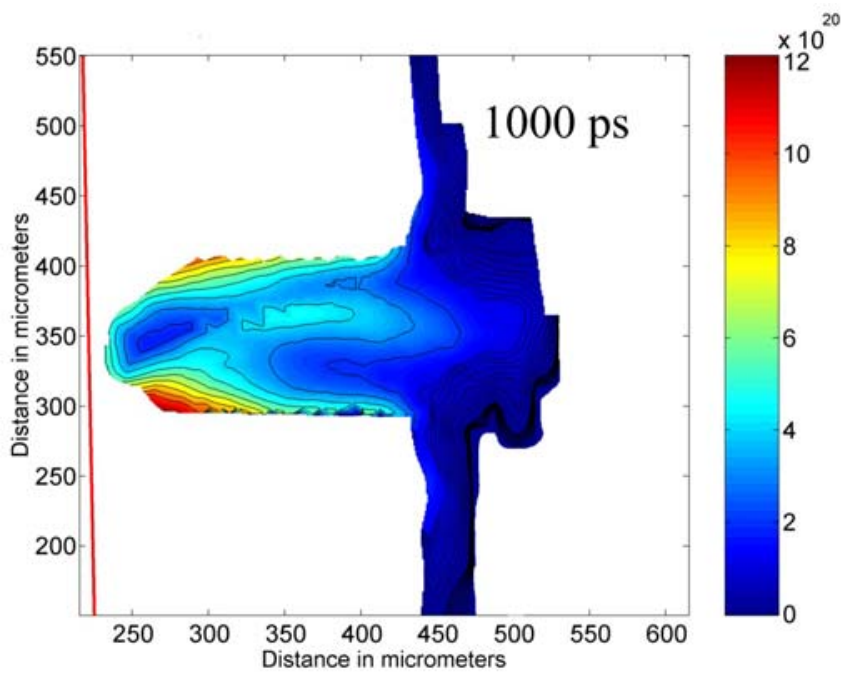

Figure 12. Electron density map corresponding to a plasma created by heating an Al groove target with the irradiation parameters of fig. 11. The interferogram was obtained $1 \mathrm{~ns}$ after the peak of the heating laser pulse. Electron densities up to $1.2 \times 10^{21} \mathrm{~cm}^{-3}$ are observed near the target walls. 


\section{Conclusions}

We have performed a series of plasma interferometry experiments with table-top soft x-ray lasers which significantly advance the goal of establishing this technique as a tool for generating detailed maps of the electron density distribution in dense plasmas. Different large-scale laser-created plasmas were studied combining a robust amplitude division interferometer based on diffraction grating beam splitters with either a portable $46.9 \mathrm{~nm}$ Ne-like Ar capillary discharge laser or a $14.7 \mathrm{~nm}$ Ni-like Pd transient soft x-ray laser. The experiments have unveiled important twodimensional effects in laser-created plasmas, which wouldn't have been clearly observed utilizing optical laser interferometry. The combination of the experimental results with hydrodynamic model calculations provides understanding of physics of these two-dimensional phenomena. The grating interferometer combines the advantages of excellent fringe visibility over a large area with high throughput and good resistance to damage by the plasma. The instrumentation and methodology we have developed is scalable to significantly shorter wavelengths, and constitutes a promising scheme for extending interferometry to the study of very dense plasmas such as those investigated for inertial confined fusion. The results demonstrate that table-top soft x-ray lasers are a powerful tool for the diagnostics of dense plasmas, that in combination with hydrodynamic codes can result in improved understanding of high density plasma phenomena

\section{Acknowledgments}

This work was supported by U.S. Department of Energy grant No. DE-FG03-02NA00062 and by the National Science Foundation. Part of this work was performed under the auspices of the U.S. Dept. of Energy by the University of California, Lawrence Livermore National Laboratory through the Institute of Laser Science and Application, under contract No. W-7405-Eng-48. The CSU researchers also gratefully acknowledge the support of the W.M. Keck Foundation.

\section{References}

1. L.B. Da Silva, T. W. Barbee, Jr. R. Cauble, P. Celliers, D. Ciarlo, S. Libby, R.A. London, D. Matthews, S. Mrowka, J.C. Moreno, D. Ress, J. E. Trebes, A. S. Wan and F. Weber, "Electron Density Measurements of High Density Plasmas Using Soft X-Ray Laser Interferometry” Phys. Rev. Lett. 74, 20, 3991 (1995).

2. P. Celliers, F. Weber, L.B. Da Silva, T. W. Barbee, Jr., R. Cauble, A. S. Wan, and J. C. Moreno, "Fringe formation and coherence of a soft-x-ray laser beam illuminating a Mach-Zehnder interferometer", Optics Letters 20, 18, 1907 (1995).

3. J. J. Rocca, C. H. Moreno, M. C. Marconi, and K. Kanizay, "Soft-x-ray laser interferometry of a plasma with a tabletop laser and a Lloyd's mirror", Optics Letters, 24, 6, 420-422 (1999).

4. C. H. Moreno, M. C. Marconi, K. Kanizay, J. J. Rocca, Yu. A. Uspenskii, A. V. Vinogradov, Yu. A. Pershin, "Softx-ray laser interferometry of a pinch discharge using a tabletop laser", Phys. Rev E. 60, 1, 911 (1999).

5. V.N. Shlyaptsev, P.V. Nickles, T. Schlegel, M.P. Kalashnikov, A.L. Osterheld, "Table-top X-ray laser pumped with subnanosecond and picosecond pulses", Proceedings of the SPIE - The Inernational Society for Optical Engineering, (Ultrashort Wavelength Lasers II, San Diego, CA, USA, 12-13 July 1993) 2012, pp. 111-18 (1993).

6. J. L. A. Chilla, J. J. Rocca, O. E. Martinez and M. . Marconi, "Soft x-ray interferometer for single-shot laser linewidth measurements", Optics Letters 21, 13, 955-957 (1996).

7. J. Filevich, K. Kanizay, M. C. Marconi, J. L. A. Chilla, J. J. Rocca "Dense plasma diagnostics with an amplitudedivision soft-x-ray laser interferometer based on diffraction gratings", Opt. Lett. 25, 5, 356-358 (2000).

8. R. F. Smith, J. Dunn, J. R. Hunter, J. Nilsen, S. Hubert, S. Jacquemot, C. Remond, R. Marmoret, M. Fajardo, P. Zeitoun, L. Vanbostal, "Longitudinal Coherence Measurement of a Transient Collisional X-ray Laser" Optics Letters (to be published).

9. G.B. Zimmerman and W.L. Kruer, Comments on Plasma Physics for Controlled Thermonuclear Fusion, 2, 51-61 (1975).

10. J.J. Rocca, E.C. Hammarsten, E. Jankowska, J. Filevich, M.C. Marconi, S. Moon, and V.N. Shlyaptsev "Application of extremely compact capillary discharge soft x-ray lasers to dense plasma diagnostics", Physics of Plasma, 10 2031-38 (2003)

11. J. Filevich, J. J. Rocca, E. Jankowska, E. C. Hammarsten, K. Kanizay, M. C. Marconi, S. J. Moon, V. N. Shlyaptsev, "Two dimensional effects in laser-created plasmas measured with soft-x-ray laser interferometry", Phys. Rev. E 67, 056409 (2003). 
12. J. Dunn, Y. Li, A. L. Osterheld, J. Nilsen, J. R. Hunter, and V. N. Shlyaptsev, "Gain Saturation Regime for LaserDriven Tabletop, Transient Ni-Like Ion X-Ray Lasers”, Phys. Rev. Lett. 84, 4834-4837 (2000).

13. R. F. Smith, J. Dunn, J. Nilsen, J. R. Hunter, V. N. Shlyaptsev, J. J. Rocca, J. Filevich, M. C. Marconi. "Refraction effects on x-ray and ultraviolet interferometric probing of laser-produced plasmas", Jour. Opt. Soc. of Am. B: Optical Physics , 20, 1, 254-259 (2003).

14. R.F. Smith, J. Dunn, J.J. Rocca,J. Nilsen, V. N. Shlyaptsev, S. Moon, J. R. Hunter, R. Shepherd, R. Booth, J. Kuba, M. Marconi. "Two Dimensional interferometric characterization of the transient collisional x-ray laser". In these Proceedings. 


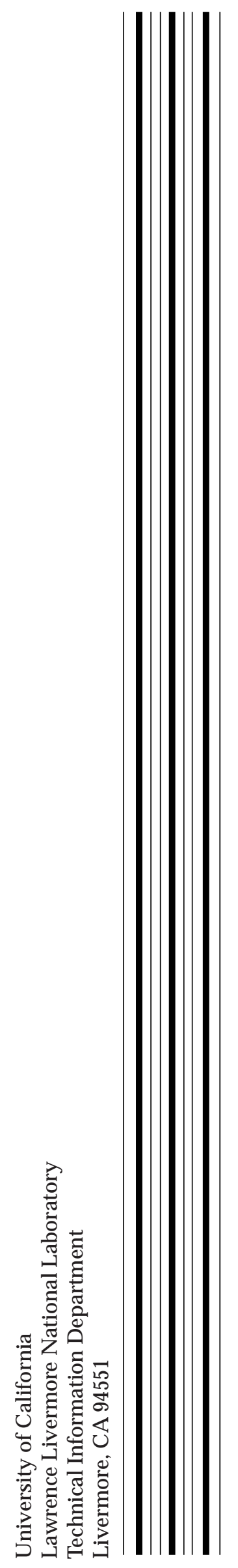

